\title{
Study of Growth and Physiological Characters in Stay-green QTL Introgression Sorghum bicolor (L.) Lines under Post-flowering Drought Stress
}

\author{
S. Sintayehu ${ }^{1}$, A. Adugna ${ }^{2,3 *}$, M. Fetene ${ }^{1}$, A. Tirfessa ${ }^{2}$ and K. Ayalew ${ }^{1}$ \\ ${ }^{1}$ Department of Plant Biology and Biodiversity Management, Addis Ababa University, \\ P.O. Box 1176, Addis Ababa, Ethiopia \\ ${ }^{2}$ Melkassa Agricultural Research Center, Adama, Ethiopia \\ ${ }^{3}$ Current address: Advanta Seeds Ltd, P.O. Box 10438, Eldoret, Kenya
}

(Received 6 January 2017; Accepted 4 July 2017;

Communicated by A. Börner)

\begin{abstract}
This experiment was carried out to evaluate the growth, physiological and yield traits of stay-green (Stg) QTL introgression sorghum lines, which were developed by the Ethiopian Institute of Agricultural Research in collaboration with ICRISAT between 2006 and 2008, under induced post-flowering drought stress. It involved a total of 12 genotypes including seven Stg QTL introgression lines, two Stg donor parents and three senescent recurrent parents. It was organized in a split plot design with three replications under well-watered and induced drought stress growing conditions at Melka Werer, Ethiopia during the post-rainy season of 2014. Analysis of variance revealed that the effect of moisture regimes on all measured traits was significant $(\mathrm{P}<0.05)$. Differences among the genotypes and genotypeby-water regime interaction were also significant $(\mathrm{P}<0.05)$ for all the traits considered. Postflowering drought stress was observed to significantly reduce most of the growth, physiological and yield related traits. The Stg introgression lines Meko/B35-selection 120, Teshale/ B35-selection 2 and Teshale/E36-1 showed better drought stress tolerance properties than the rest of the genotypes based on the measured growth and physiological traits. These introgression lines also showed better grain yield than their recurrent parents under post-flowering drought stress and can be used as new versions of the existing varieties (served as recurrent parents) and for future breeding programs. Furthermore, leaf chlorophyll content, assimilation rate, transpiration rate, water use efficiency, root length and root dry weight were found to have strong correlation and can be used to screen genotypes for post-flowering drought tolerance.
\end{abstract}

Keywords: drought, growth, QTL introgression lines, sorghum, stay-green

\section{Introduction}

Sorghum [Sorghum bicolor (L.) Moench], that has been evolved in the semi-arid tropical Africa where it is still used as a major food grain is the fifth important cereal worldwide. It is cultivated as a major crop in areas with marginal growing conditions such as poor soil

\footnotetext{
*Corresponding author; E-mail: asfawadugna@yahoo.com
} 
fertility and erratic rainfall (Bibi et al. 2012). Ethiopia is the third largest producer of sorghum in Africa after Nigeria and Sudan. In Ethiopia, sorghum is the third most important cereal next to teff and maize both in cultivated area and in total grain production (Adugna 2007).

Drought reduces production of sorghum crop and risks the wellbeing, livelihood, as well as food security in many developing countries where it serves as a staple (Dial 2012). Its effect starts from seed germination and continues until grain filling by disturbing morphological, physiological, and biochemical processes of crops (Bibi et al. 2012). Drought disrupts the physiology of plants by reducing water content, causing wilting, closing of stomata, and decreasing cell enlargement and growth (Taiz and Zeiger 2006). It restricts plant growth, both in terms of the total quantity of tissue produced and the time that the plant tissue is produced (Farooq et al. 2009). Leaf expansion and root elongation are the most water deficit sensitive turgor-dependent activities as a result of cell and cell wall shrinking (Taiz and Zeiger 2006). Moreover, water deficit results in decreasing leaf expansion and leaf area which results from biophysical effect of turgor pressure (Taiz and Zeiger 2006; Rostampour et al. 2012). The effect of drought depends up on its timing of occurrence, duration and intensity. When drought stress progresses, it can accelerate leaf senescence and lead to death of leaf tissue (Prasad et al. 2008).

Sorghum genotypes respond differently to pre-flowering and post-flowering drought stresses. Understanding the physiological mechanisms of plant water stress response is necessary to breed drought tolerant sorghum. For instance drought is known to decrease relative water content and water potential (Kamran et al. 2014). In sorghum, post-flowering drought stress reduces photosynthesis due to stomatal and non-stomatal limitations (Taiz and Zeiger 2006; Mafakheri et al. 2010). Enhancement of sorghum genotypes tolerant to drought ensures better yield, resistance to lodging and charcoal rot (Reddy et al. 2014). Stay-green trait is considered as post-flowering drought tolerance mechanism in sorghum, which enables plants to remain green and fill grain normally (Rosenow et al. 1983). It also contributes to increased grain yield due to increased grain size and number (Borrell et al. 1999). QTLs have been mapped for the Stg trait in sorghum (Xu et al. 2000) and have been used for marker assisted breeding (Adugna and Tirfessa 2014). Therefore, the objectives of this study were: 1) To evaluate variations among stay-green sorghum introgression lines and their parents for growth, physiological, yield and yield related traits under post-flowering drought stress. 2) To identify and suggest genotypes that could be used in areas prone to post-flowering drought stress, and 3) to identify the best growth and physiological adaptive traits that can be helpful for future evaluation of sorghum genotypes under post-flowering drought stress.

\section{Materials and Methods}

\section{Description of the study area}

The experiment was conducted during the post-rainy season (February-June) of 2014 at Melka Werer Agricultural Research Center, Ethiopia $\left(40^{\circ} 11^{\prime} \mathrm{E}, 9^{\circ} 22^{\prime} \mathrm{N}\right.$, and altitude 750 $\mathrm{m})$. Melka Werer is a semi-arid, drought prone area and preferred for the study because 
the historical weather data showed little rainfall during the study period. Moreover, the research center has well-organized irrigation facility.

\section{Experimental materials}

A marker assisted Stg QTL introgression was carried out by the national sorghum research program of the Ethiopian Institute of Agricultural Research in collaboration with ICRISAT, Kenya between 2006 and 2008. This study evaluated a total of 12 genotypes including seven Stg sorghum introgression lines derived through marker assisted backcrossing (Meko/B35-selection 120, Meko/B35-selection 116, Meko/B35-selection 117, Teshale/E36-1, Teshale/B35-selection 1, Teshale/B35-selection 2, Gambella/B35), three of their senescent recurrent parents (Meko, Teshale and Gambella 1107) and the two Stg donor parents, B35 and E36-1. The Stg introgression lines were at the stage of $\mathrm{BC}_{3} \mathrm{~F}_{4}$. These genotypes were obtained from the Ethiopian sorghum research program of the Ethiopian Institute of Agricultural Research (EIAR) at Melkassa Agricultural Research Center, Ethiopia. The recurrent parents are early maturing and high yielding varieties, which were released for drought prone lowland environments of Ethiopia, but they have been affected by terminal drought. The Stg donor parents were introduced from ICRISAT and they were derivatives of accessions originally obtained from Ethiopia. The details of the introgression process are available in Adugna and Tirfessa (2014).

\section{Experimental design and treatments}

The field experiment was laid out in split plot design with three replications. The main plot factors were two moisture regimes: well-watered and induced drought stress; and the sub-plot factors were the introgression lines and their donor and recurrent parents (hereafter referred to as genotypes). The seeds were drilled manually into a plot of three rows of $3.5 \mathrm{~m}$ length with spacing of $0.75 \mathrm{~m}$. Two weeks after sowing, the seedlings were thinned to an interplant spacing of $0.15 \mathrm{~m}$. Fertilizers were applied at the recommended rates of $100 \mathrm{~kg} \mathrm{ha}^{-1}$ of diammonium phosphate (DAP; $18 \% \mathrm{~N}$ and $46 \% \mathrm{P}_{2} \mathrm{O}_{5}$ ) in the seed furrows during sowing and $50 \mathrm{~kg} \mathrm{ha}^{-1}$ urea $(46 \% \mathrm{~N})$ a split application where half of it was applied during sowing and the remaining top dressed at the six leaf stage. All recommended management practices (weeding, cultivation, etc.) were applied uniformly to all the plots. The two contrasting soil moisture regimes were created through varying the frequency of irrigation after flowering was completed using furrow irrigation method. For both growing conditions, the field was equally irrigated from planting until flowering. However, post-flowering drought stressed condition was created after flowering was completed by withholding irrigation water. On the contrary, the well-watered treatments continued receiving irrigation water until maturity. Thus, the well-watered treatments received four times more irrigation water after flowering than the drought stressed treatments. 


\section{Data recording}

\section{Growth and phenological characters}

Plant height (PH), leaf area (LA), Stg score (SGS), and days to physiological maturity (DTM) were considered as growth and phenological characters and were recorded as per the descriptors for sorghum (IBPGR/ICRISAT 1993). Leaf area was calculated as leaf length $\times$ leaf width $\times 0.747$ (the shape factor) (Sticker et al. 1961). Visual Stg ratings were taken at physiological maturity to determine if drought induction caused leaf and whole plant death in a plot. One to five scoring were adopted as scales based on the proportion of LA of normal sized leaves that remained green, had prematurely senesced or died. A rating 1 indicates completely green normal sized leaves (no leaf death), $2=25 \%$ of the leaves died, $3=26$ to $50 \%$ of the leaves died, $4=51$ to $75 \%$ were dead, $5=76$ to $100 \%$ of the leaves and stalk were dead (complete plant death). The DTM was recorded as the number of days from sowing until $95 \%$ of the plants in a plot formed black hilar layer.

\section{Physiological characters}

Leaf chlorophyll content (LCC), assimilation rate (AR), transpiration rate (TR), water use efficiency (WUE), and relative water content (RWC), were measured as physiological characters. Leaf chlorophyll content was measured at physiological maturity from five randomly selected and tagged plants per plot from two leaves per plant using a CCM-200 pulse chlorophyll content meter (ADC BioScientific Ltd, UK). Water use efficiency was determined by taking the ratio of AR to water lost due to transpiration. To measure RWC, flag leaves were collected randomly from five plants in each plot, leaf discs of area $2 \mathrm{~cm}^{2}$ excised from each leaf and fresh weight (FW) measured. They were placed in distilled water for $24 \mathrm{~h}$ and reweighed to obtain turgid weight (TW). They were, then oven dried at $70{ }^{\circ} \mathrm{C}$ for $24 \mathrm{~h}$ followed by root dry weight measurement. Relative water content was calculated as per Barrs (1968). Net photosynthesis and TR per unit area were measured as gas exchange parameters using portable photosynthesis system LCpro+ (ADC BioScientific Ltd, UK) from the $2^{\text {nd }}$ and $3^{\text {rd }}$ intact leaves from the top of each plant. The data were recorded 30 days after post-flowering drought has been inducted in the morning from 07:00AM to 10:00AM.

\section{Determination of root dry weight, yield and yield components}

After harvest, observations were recorded on root length (RL) and root dry weight (RDW). To measure RDW, three sample plants were uprooted from each plot by excavating carefully not to damage the roots. The shoot and root systems were separated carefully and the roots were washed with tap water gently until the soils were completely removed. The roots were kept in an oven at $70{ }^{\circ} \mathrm{C}$ for $72 \mathrm{~h}$ and root dry weight (g/plant) was recorded using sensitive electronic balance. Panicle weight was recorded on oven dried panicles. Grain yield (GY) per plot was measured in grams, which was later converted into kilograms per hectare. The weight of hundred grains (HSW) was also recorded. 


\section{Statistical analysis}

All the collected data were subjected to analysis of variance (ANOVA) using STATISTICA Software Version 7 (STATISTICA Inc., USA) to observe variations among sorghum genotypes, between the two water regimes, and genotype-by-irrigation interaction. The genotype means were separated using the Tukey's HSD test. Moreover, the correlations among the studied traits were analyzed.

\section{Results}

Analysis of variance revealed significant differences $(\mathrm{P}<0.05)$ among the genotypes, between the two water regimes, and genotype-by-irrigation interaction for all the measured growth, phenological, physiological, root and yield related traits (data not shown), which indicates that the studied genotypes were diverse and their response to the two water regimes was different.

\section{Growth and phenological traits}

Leaf area showed decreasing trend under drought stress, but the extent of the reduction was higher in the senescent parents Meko and Gambella and less in Meko/B35-selection 120 and Teshale/E36-1. Under drought stress, all the introgression lines of Teshale had larger LA than Teshale, but Gambella1107 and Meko had larger LA than their introgression lines Gambella1107/B35, Meko/B35-selection 116 and Meko/B35-selection 117. As time of exposure to drought stress progressed, plants showed a sharp decline in LA compared to that in well-watered condition. The average DTM was shortened by about five days due to post-flowering drought stress. As expected, the Stg donor parents, E36-1 and B35, and two of their introgression lines, Meko/B35-selection 120 and Meko/B35-selection 116 maintained their green leaves until maturity. Drought had significantly hastened leaf senescence (SGS), but the rate was lower in those genotypes with Stg QTLs (Stg donors and introgression lines) and higher in the recurrent parents (Table 1).

\section{Physiological characters}

Under drought stress, introgression lines and their donor parents maintained higher LCC until physiological maturity than that in the recurrent parents. Accordingly, the two donor parents, B35 and E36-1 had LCC of 54.45 and $52.76 \mu \mathrm{g} / \mathrm{cm}^{2}$, respectively. The LCC of the recurrent parents Gambella 1107 and Meko reduced from 36.26 to $23.85 \mu \mathrm{g} / \mathrm{cm}^{2}$ and from 36.26 to $21.63 \mu \mathrm{g} / \mathrm{cm}^{2}$, respectively (Table 1). Drought stress drastically reduced AR of all the genotypes, but still the introgression lines had advantages over their recurrent parents. B35 and E36-1, had the highest AR of all the genotypes. The lines, Meko/B35selection 120, Teshale/B35-selection 2, Teshale/E36-1, and Meko/B35-selection 116 had higher AR than their recurrent parents. The TR of genotypes under stress was lower than that under well-watered condition. The highest TR was observed in Stg donor parents. 


\begin{tabular}{|c|c|c|c|c|c|c|c|c|c|c|c|c|}
\hline$\sum_{3}^{\mid 山}$ & 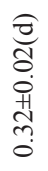 & 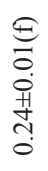 & 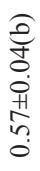 & 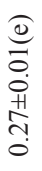 & 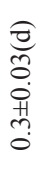 & 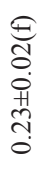 & 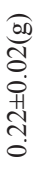 & 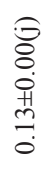 & $\begin{array}{l}\mathbb{3} \\
\mathbb{0} \\
0 \\
0 \\
0 \\
0 \\
0 \\
0 \\
0 \\
0\end{array}$ & 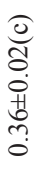 & 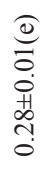 & 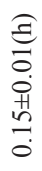 \\
\hline 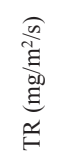 & 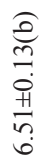 & 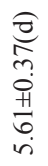 & 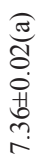 & 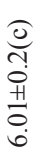 & 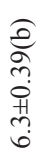 & $\begin{array}{l}\hat{0} \\
6 \\
0 \\
+ \\
+1 \\
n \\
i n\end{array}$ & 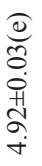 & 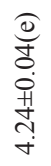 & 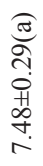 & 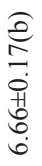 & 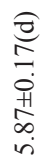 & 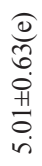 \\
\hline 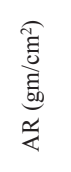 & 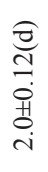 & 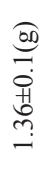 & 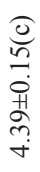 & 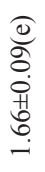 & 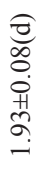 & 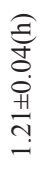 & 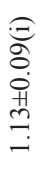 & 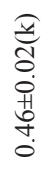 & 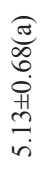 & 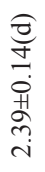 & 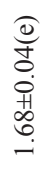 & 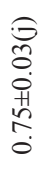 \\
\hline 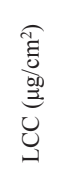 & 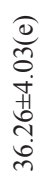 & 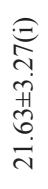 & 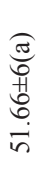 & 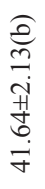 & 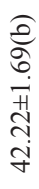 & $\begin{array}{l}\stackrel{E}{m} \\
m \\
\stackrel{m}{\#} \\
\frac{\vec{n}}{m}\end{array}$ & 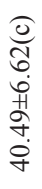 & 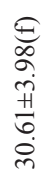 & 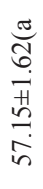 & 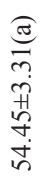 & 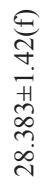 & 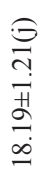 \\
\hline స్ & 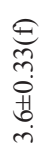 & 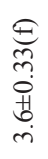 & 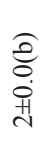 & 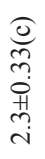 & 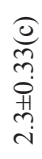 & $\begin{array}{l}\text { () } \\
\text { m } \\
\text { o } \\
\text { m }\end{array}$ & 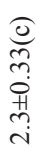 & $\begin{array}{l}\text { (e) } \\
\text { ñ } \\
\text { i⿱ } \\
\text { m }\end{array}$ & $\begin{array}{l}\overparen{\Xi} \\
\stackrel{0}{0} \\
\text { \# }\end{array}$ & 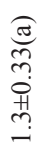 & 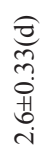 & 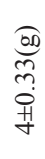 \\
\hline 胥 & 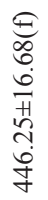 & 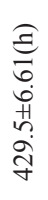 & 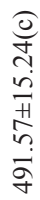 & 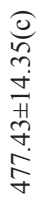 & 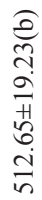 & 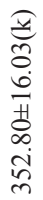 & 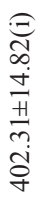 & 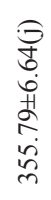 & 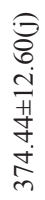 & 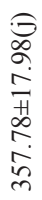 & 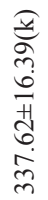 & 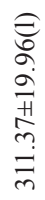 \\
\hline $\begin{array}{l}\widehat{\Xi} \\
\text { 吾 }\end{array}$ & 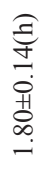 & 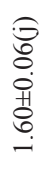 & 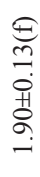 & 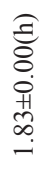 & 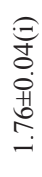 & 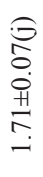 & 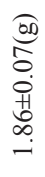 & 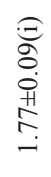 & 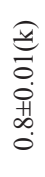 & 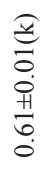 & 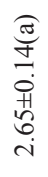 & 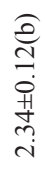 \\
\hline 总 & 3 & $\tilde{\Omega}$ & 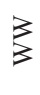 & $\tilde{n}$ & 3 & $\tilde{\rho}$ & 3 & $\tilde{\Omega}$ & 3 & $\tilde{\Omega}$ & 3 & $\tilde{\Delta}$ \\
\hline 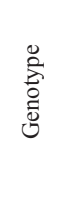 & \multicolumn{2}{|c|}{ 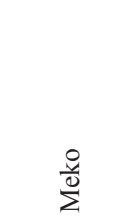 } & \multicolumn{2}{|c|}{ 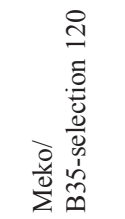 } & \multicolumn{2}{|c|}{  } & \multicolumn{2}{|c|}{ 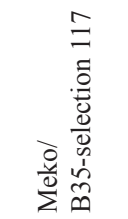 } & & \multicolumn{2}{|c|}{ 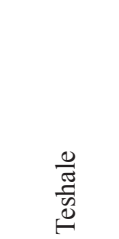 } \\
\hline
\end{tabular}




\begin{tabular}{|c|c|c|c|c|c|c|c|c|c|c|c|c|}
\hline$\sum_{3}^{m}$ & 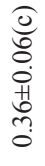 & 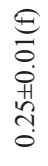 & 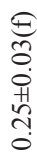 & 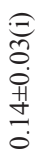 & $\begin{array}{l}0 \\
0 \\
0 \\
0 \\
0 \\
+1 \\
m \\
0\end{array}$ & 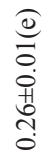 & 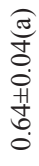 & 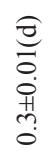 & 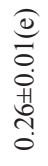 & 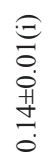 & 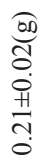 & 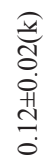 \\
\hline 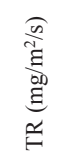 & 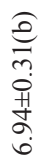 & 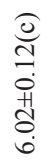 & 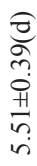 & $\begin{array}{l}\text { () } \\
\text { nn } \\
0 \\
0 \\
n \\
r \\
\dot{r}\end{array}$ & 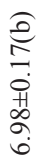 & 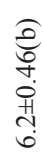 & 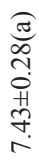 & 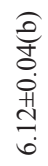 & $\begin{array}{l}\text { t) } \\
n \\
0 \\
+1 \\
n \\
i n\end{array}$ & $\begin{array}{l}\text { () } \\
\stackrel{+}{+} \\
\dot{+} \\
\text { مे } \\
\dot{+}\end{array}$ & 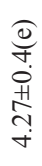 & 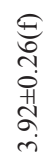 \\
\hline 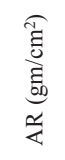 & 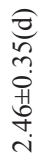 & 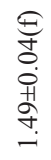 & 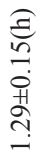 & 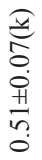 & 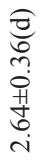 & 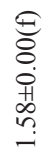 & 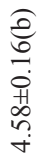 & 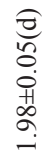 & 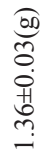 & $\begin{array}{l}\text { Oे } \\
0 \\
0 \\
0 \\
\text { H. } \\
0 \\
0\end{array}$ & 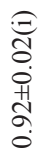 & 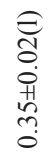 \\
\hline 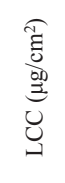 & 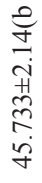 & 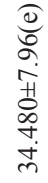 & 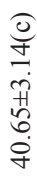 & 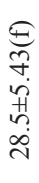 & $\begin{array}{l}\hat{0} \\
0 \\
\infty \\
i \\
\text { त } \\
\infty \\
o \\
0 \\
+\end{array}$ & 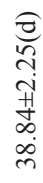 & $\begin{array}{l}\cong \\
\stackrel{\Xi}{n} \\
+1 \\
n \\
n \\
n \\
n\end{array}$ & 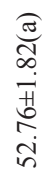 & 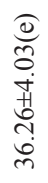 & 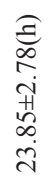 & $\begin{array}{l}\text { (0) } \\
n \\
n \\
\text { त } \\
n \\
0 \\
0\end{array}$ & 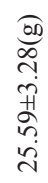 \\
\hline 包 & 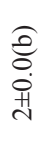 & 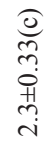 & 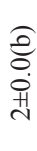 & $\begin{array}{l}\text { है } \\
\text { m. } \\
\text { Oे } \\
\text { t. } \\
\text { i }\end{array}$ & $\begin{array}{l}\hat{\theta} \\
0 \\
0 \\
\text { H } \\
\text { N }\end{array}$ & 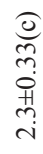 & 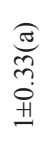 & 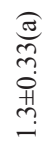 & 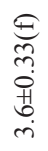 & $\begin{array}{l}\text { On } \\
\text { D0 } \\
0 \\
0 \\
\text { m } \\
+\end{array}$ & 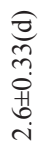 & $\begin{array}{l}\text { (0) } \\
\text { Oे } \\
\dot{0} \\
\text { H }\end{array}$ \\
\hline $\begin{array}{l}\text { हु } \\
\text { ङ }\end{array}$ & 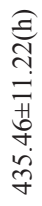 & 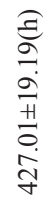 & 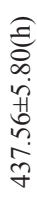 & 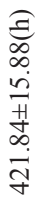 & 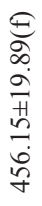 & 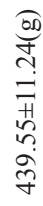 & 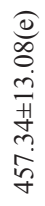 & 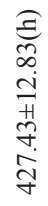 & 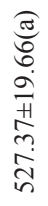 & 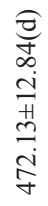 & 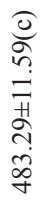 & 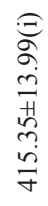 \\
\hline $\begin{array}{l}\widehat{\Xi} \\
\text { 吾 }\end{array}$ & 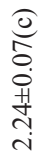 & 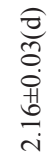 & 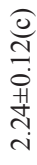 & 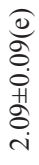 & 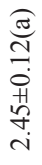 & 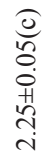 & 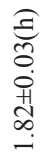 & $\begin{array}{l}\overparen{\Xi} \\
\stackrel{0}{0} \\
\text { H } \\
\stackrel{H}{2}\end{array}$ & 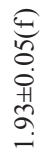 & 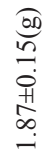 & 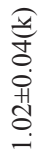 & 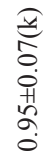 \\
\hline 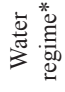 & 3 & $\tilde{\Delta}$ & 3 & $\tilde{\Lambda}$ & 3 & $\tilde{\rho}$ & 3 & $\tilde{A}$ & 3 & $\tilde{a}$ & $\sum_{3}^{3}$ & $\tilde{\Omega}$ \\
\hline 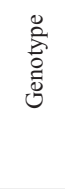 & & \multicolumn{2}{|c|}{ 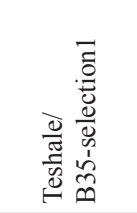 } & \multicolumn{2}{|c|}{ 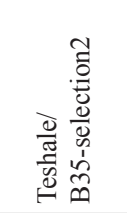 } & & \multicolumn{2}{|c|}{ 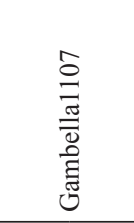 } & \multicolumn{2}{|c|}{ 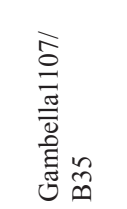 } \\
\hline
\end{tabular}




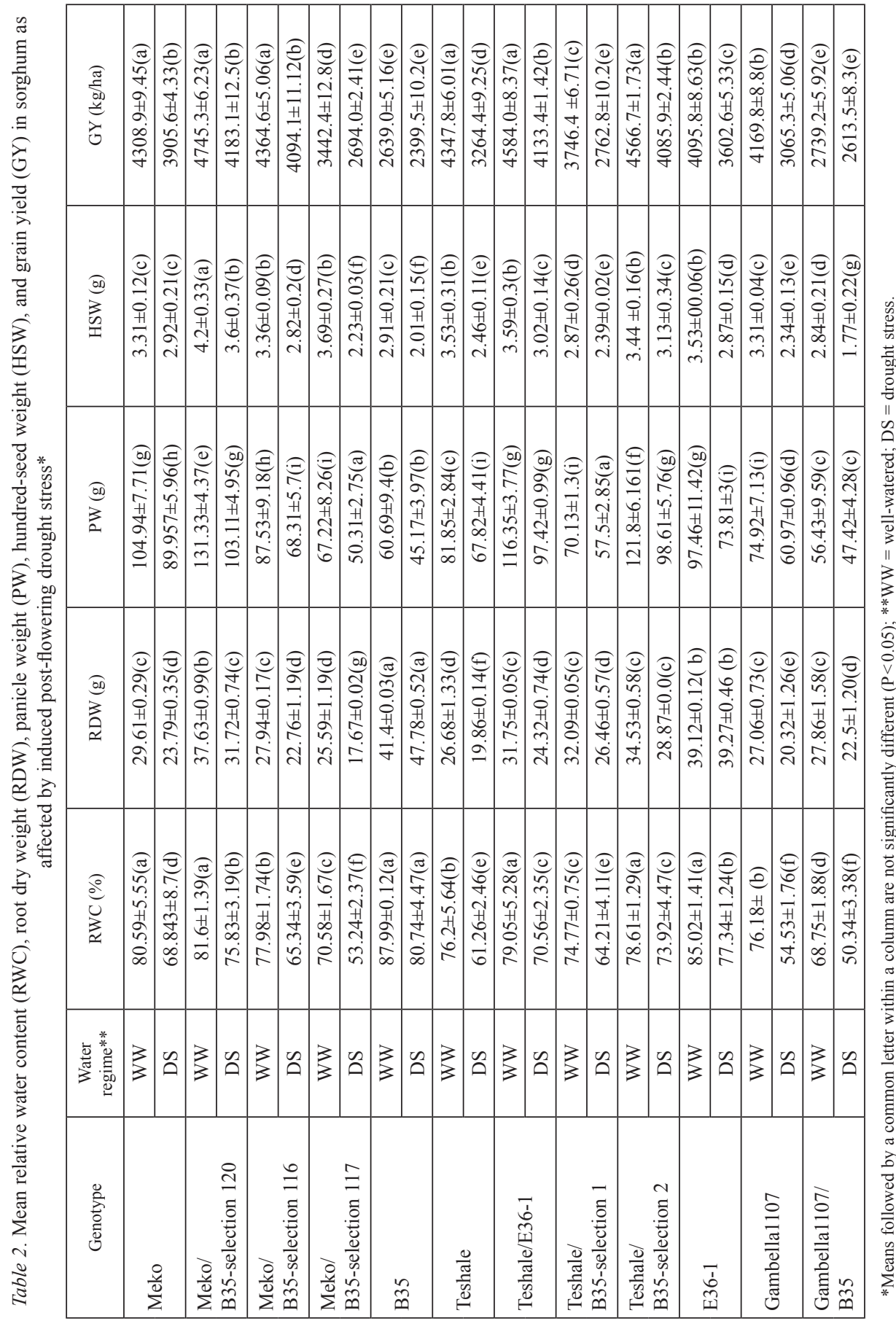


The introgression lines Teshale/B35-selection 2, Teshale/E36-1, and Meko/B35-selection 120 also had higher TR than their recurrent parents. TR in Gambella 1107/B35 and Gambella 1107 was not affected by drought. WUE of genotypes under drought stress condition ranged from 0.12 (Gambella 1107/B35) to 0.36 (B35). Meko/B35-selection 120, Teshale/E36-1, and Teshale/B35-selec 2 showed better WUE than their recurrent parents (Table 1). The introgression lines Teshale/B35-selection 2 and Teshale/E36-1 had higher RWC than their recurrent parent, Teshale. In addition, Meko/B35-selection 120 had higher RWC than the lines, Meko/B35-selection 116 and Meko/B35-selection 117, which had different Stg QTLs. The introgression line Gambella1107/B35 had the lowest RWC of all the genotypes under stress (Table 2).

\section{Root dry weight, grain yield and yield related traits}

Genotypes showed significant variation in RDW in response to the two water levels. The Stg donor parents had higher RDW than the non-Stg recurrent parents and the Stg introgression lines both under well-watered and drought stress. The introgression lines, Meko/ B35-selection 120, Teshale/B35-selection 1, Teshale/B35-selection 2, Teshale/E36-1, and Gambella1107/B35 had higher RDW than their recurrent parents under stress (Table 2).

Under drought stress, Meko/B35-selection 120 attained the highest HSW and PW of all the genotypes tested including its recurrent parent. Teshale/B35-selection 1 and Teshale/E36-1 had higher HSW than their recurrent parent, Teshale. Moreover, Meko/B35selection 120, Teshale/B35-selection 2, and Teshale/E36-1 had higher PW than their respective recurrent parents, Meko and Teshale. Reduction in GY was observed in all the genotypes under stress. The average GY of all the genotypes was $3979.15 \mathrm{~kg} \mathrm{ha}^{-1}$ and $3400.35 \mathrm{~kg} \mathrm{ha}^{-1}$ for well-watered and drought stress, respectively. The introgression lines Meko/B35-selection 116, Meko/B35-selection 120, Teshale/B35-selection 2, Teshale/ E36-1, had higher GY than their recurrent parents (Table 2).

\section{Correlations among characters}

In conditions of post-flowering drought stress, LA had little or no correlation with the rest of the characters measured. LCC had significant and positive correlation with RWC $(\mathrm{r}=0.62)$, AR $(r=0.73)$, TR $(r=0.48)$, WUE $(r=0.71)$, RDW $(r=0.80)$ and AR was significantly and positively correlated with LCC, TR $(r=0.80)$, WUE $(r=0.95)$, RWC $(\mathrm{r}=0.78)$, RDW $(\mathrm{r}=0.83)$, RL $(\mathrm{r}=0.34)$, HSW $(\mathrm{r}=0.36)$ and PW $(\mathrm{r}=0.44)$. However, only RL was significantly correlated with GY (Table S1*).

\section{Discussion}

\section{Growth and phenological traits}

Leaf area during grain filling is considered to be the most important character to determine grain yield under drought stress condition. In this study, post-flowering drought has

*Further details about the Electronic Supplementary Material (ESM) can be found at the end of the article. 
decreased the mean LA of all the genotypes by about $10 \%$, but the magnitude was the highest in Meko/B35-selection 116 and the lowest in Teshale/E36-1. Adugna and Tirfessa (2014) also reported similar reduction in LA.

Post-flowering drought stress has resulted in decline of the proportion of green leaves in recurrent parents, while the Stg donor lines and some of the introgression lines retained larger number of green leaves until maturity. Similar results were reported by Kassahun et al. (2010) and was attributed to the contribution of Stg to longevity of leaves (SooCheul et al. 2007). The results of Stg rating under exposure to post-flowering drought showed that some of the recurrent parents and their introgression lines senesced faster than others, which was in agreement with Soo-Cheul et al. (2007), which might be due to a series of biochemical and physiological process, which further degraded photosynthetic apparatus (Campanile et al. 2000). Shortening of DTM in Teshale, Gambella 1107/B35 and Meko/B35-selection 117, was in agreement with the results of Bezabih (2012) and Adugna and Tirfessa (2014) and could be due to forced maturity as a result of induced drought stress. Panicle weight and HSW were found to have significant positive correlation with GY in conditions of drought.

\section{Physiological traits}

In different studies, determination of LCC has been used as a screening tool for selection of drought tolerant genotypes (Malala 2010) as exposure to drought stress leads to a significant decrease in Chlorophyll $a$ and Chlorophyll $b$ contents (Talebi et al. 2013). In this study, drought has significantly reduced LCC in senescent recurrent parents Teshale, Meko and Gambella1107, which may be due to the degradation of leaf proteins that are bound to chlorophyll pigment-protein complex and lower $\mathrm{N}$ absorption during the grain filling period (Borrell and Hammer 2000; Borrell et al. 2001). On the other hand, genotypes possessing Stg were reported to have the capacity to maintain high LCC (Soo-Cheul et al. 2007). In this study as well, all of the introgression lines had higher LCC than their recurrent parents. The present study showed that drought stress significantly reduced photosynthesis in some of the tested introgression lines, which had lower AR. This was in conformation with the results of some of the previous reports (e.g. Khakwani et al. 2012) and could be due to stomatal closure as a consequence of lower water content or hormonal (ABA) signals transferred to the leaves, which in turn might have affected AR (Khakwani et al. 2012). Genotypes Meko/B35-selection 120, Teshale/B35-selection 2 and Teshale/ E36-1, which had larger LA also had better AR. The opposite was true for genotypes, which had smaller LA. This was in agreement with Blum (2005) and could be as a result of decreased green leaves, photosynthetic active radiation (PAR) absorption of antenna complexes, WUE and due to degradation of chlorophyll (Borrell et al. 2001).

Measuring RWC is useful indicator of plant water balance and water potential parameter under drought stress conditions (Kamran et al. 2014) as it conveys the relative amount of water present on the plant tissues (Yamasaki and Dillenburg 1999). Different studies have shown significant difference among genotypes for RWC when grown under drought stress condition (Abdullah et al. 2011; Hasheminasab et al. 2012). In the present study, all 
of Teshale derived introgression lines and Meko/B35-selection 120 had better RWC than their recurrent parents. This might be due to lower accumulation of osmolytes to maintain tissue turgor (Abdullah et al. 2011). In addition, Meko/B35-selection 120 had higher RWC than lines Meko/B35-selection 116 and Meko/B35-selection 117, perhaps due to the differences in the introgressed QTLs. The Stg introgression lines, Meko/B35-selection 120, Teshale/E36-1, and Teshale/B35-selection 2 had higher WUE than their recurrent parents, which was in agreement with the findings of Thevar et al. (2010), and was perhaps due to increased AR and TR as WUE is the ratio of whole-plant biomass to cumulative transpiration (Kapanigowda et al. 2012). It could also be due to higher RDW (Songsri et al. 2009), which increased water absorption (Thevar et al. 2010).

\section{Root dry matter yield, grain yield and yield components}

High root biomass contributes to high absorption efficiency, which affords the cost of transpiration and increased WUE and transpiration (Thevar et al. 2010). The introgression lines derived from Teshale, Meko/B35-selection 120 and Gambella 1107/B35 had higher RDW than their recurrent parents. The present study has shown that RDW was positively correlated with LCC, AR, TR, WUE and RWC. Under lower soil moisture, high root biomass ensures better absorption, which further contributes to high yield production (Blum 2005), but in this study only RL had significant positive correlation with GY. In drought tolerant genotypes, root traits are reportedly positively correlated with GY as high water extracting ability of a plant leads to more photosynthetic activity, photo assimilate and high partitioning, which in turn lead to increased GY (Atta et al. 2013). Compared to preflowering drought stress, post-flowering drought stress reportedly significantly decreases GY due to the failure in pollen fertility and improper grain filling (Shamsi et al. 2010). The present study has shown that drought reduced GY in all of the tested genotypes, but the extent was the highest in the recurrent parents Gambella 1107 (26\%) and Teshale $(25 \%)$ and the introgression line Teshale/B35-selection 1, while the lowest in Gambella $1107 / \mathrm{B} 35$ (4.6\%). The genotype mean GY was reduced by $15.5 \%$ as a result of drought. The introgression lines Meko/B35-selection 116, Meko/B35-selection 120, Teshale/E361 and Teshale/B35-selection 2 had better GY than their recurrent parents under drought stress. Panicle weight and HSW had significant positive correlation with GY, which was in agreement with previous reports (e.g. Malala 2010).

In summary, the present study has revealed varied responses to post-flowering drought in sorghum genotypes. Out of the introgression lines, Meko/B35-selection 120, Teshale/ B35-selection 2 and Teshale/E36-1 showed consistently better drought tolerance than their recurrent parents as revealed by the different growth, physiological, root, yield and yield related measurements. Therefore, these lines could be used as new versions of the recurrent parents (existing varieties) and for further breeding work. On the other hand, though Meko/B35-selection 116 had better yield than Meko, its drought tolerance was not confirmed by the different measurements. Similarly, though Teshale/B35-selection 1 had better drought tolerance as revealed by the different measurements, its GY was inferior to Teshale, the recurrent parent. Among the measurements, LCC, TR, AR, WUE, RWC, RL 
and RDW mostly had positive association with post flowering drought tolerance of the genotypes. Even though positive results were found using the studied characters, it is recommended that future biochemical studies be included to have a comprehensive understanding of the mechanisms associated with drought tolerance in sorghum.

\section{Acknowledgements}

We thank the national sorghum research program of the Ethiopian Institute of Agricultural Research (EIAR) at Melkassa Agricultural Research Center, Adama for providing with the experimental materials. The Department of Plant Biology and Biodiversity Management of Addis Ababa University is also hereby acknowledged for the laboratory space and equipment.

\section{References}

Abdullah, F., Hareri, F., Naaesan, M., Ammar, M.A., Kanbar, O.Z. 2011. Effect of drought on different physiological characters and yield component in different varieties of Syrian durum wheat. J. of Agric. Sci. 3:127-133.

Adugna, A. 2007. The role of introduced sorghum and millets in Ethiopian agriculture. SAT e-Journal 3:1-4.

Adugna, A., Tirfessa, A. 2014. Response of stay-green quantitative trait locus (QTL) introgression sorghum lines to post-anthesis drought stress. African J. of Biotechnol. 13:4492-4500.

Atta, B.M., Mahmood, T., Trethowan, R.M. 2013. Relationship between root morphology and grain yield of wheat in north-western NSW, Australia. Aust. J. of Crop Sci. 7:2108-2115.

Barrs, H.D. 1968. Determination of water deficits in plant tissue. In: Kozlowski, T.T. (ed.) Water Deficits and Plant Growth. Academic Press. New York, USA. vol. 1, pp. 235-368.

Bezabih, A. 2012. Evaluation of stay-green quantitative trait loci (QTLs) introgression sorghum (Sorghum bicolor (L.) Moench) lines for post-flowering drought resistance at Kobo, North eastern Ethiopia. M.Sc. Thesis, Haramaya University. Alemaya, Ethiopia.

Bibi, A., Sadaqat, H.A., Tahir, M.H.N., Akram, H.M. 2012. Screening of sorghum (Sorghum bicolor var Moench) for drought tolerance at seedling stage in polyethylene glycol. The J. of Animal and Plant Sci. 22:671-678.

Blum, A. 2005. Drought resistance, water-use efficiency, and yield potential are they compatible, dissonant, or mutually exclusive? Aust. J. of Agric. Res. 56:1159-1168.

Borrell, A., Hammer, G., Van Oosterom, E. 2001. Stay-green: A consequence of the balance between supply and demand for nitrogen during grain filling? Ann. Appl. Biol. 138:91-95.

Borrell, A.K., Bidinger, F.R., Sunitha, K. 1999. Stay-green associated with yield in recombinant inbred sorghum lines at varying rate of leaf senescence. ISML 40:31-33.

Borrell, A.K., Hammer, G.L. 2000. Nitrogen dynamics and the physiological basis of stay-green in sorghum. Crop Sci. 40:1295-1307.

Campanile, R.G., DeVita, P., DiFonzo, N., Flagella, Z., Laus, M., Lawlor, D., Padalino, L., Rascio, A., Russo, M., Shewry, P.R., Spano, G., Stoppelli, M.C., Troccoli, A., Trono, D. 2000. Resistance to a biotic stresses in durum wheat: Which ideotype? In: Royo, C., Nachit, M., DiFonzo, N., Araus, J.L. (eds), Durum Wheat Improvement in the Mediterranean Region: New Challenges. CIHEAM. Zaragoza, Spain. pp. 215-225.

Dial, H.L. 2012. Plant Guide for Sorghum (Sorghum bicolor L.). USDA-Natural Resources Conservation Service, Tucson Plant Materials Center. Tucson, AZ, USA.

Farooq, M., Wahid, A., Kobayashi, N., Fujita, D., Basra, S.M.A. 2009. Plant drought stress: effects mechanisms and management. Agronomy for Sustainable Devel. 29:185-212.

Hasheminasab, H., Assad, M.T., Aliakbari, A., Sahhafi, S.R. 2012. Evaluation of some physiological traits associated with improved drought tolerance in Iranian wheat. Ann. of Biol. Res. 3:1719-1725.

IBPGR and ICRISAT. 1993. Descriptors for sorghum [Sorghum bicolor (L.) Moench]. International Board of Plant Genetic Resources, Rome, Italy. ICRISAT, Patacheru, India. 
Kamran, M., Naeem, M.K., Ahmad, M., Nawazshah, M.K., Iqbal, M.S. 2014. Physiological responses of wheat (Triticum aestivum L.) against drought stress. Am. J. Res. Commun. pp. 1-9. www.usa-journals.com. ISSN:2325-4076.

Kapanigowda, M.H., Payne, W.A., Rooney, W.L., Mullet, J.E. 2012. Transpiration ratio in sorghum [Sorghum bicolor (L.) Moench] for increased water-use efficiency and drought tolerance. J. of Arid Land Studies 22:175-178.

Kassahun, B., Bidinger, F.R., Hash, C.T. 2010. Stay-green expression in early generation sorghum (Sorghum bicolor (L.) Moench) QTL introgression lines. Euphytica 172:351-362.

Khakwani, A.A., Dennett, M.D., Munir, M., Baloch, M.S. 2012. Wheat yield response to physiological limitations under water stress condition drought resistance mechanisms. The J. of Animal and Plant Sci. 22:773-780.

Mafakheri, A., Siosemardeh, B., Bahramnejad, B., Struik, P.C., Sohrab, Y. 2010. Effect of drought stress on yield, proline and chlorophyll contents in three chickpea cultivars. Aust. J. of Crop Sci. 4:580-585.

Malala, T.J. 2010. Evaluation and selection of 20 Sorghum (Sorghum bicolor (L.) Moench) genotypes for drought tolerance. M.Sc. Thesis, University of Pretoria. Pretoria, South-Africa.

Prasad, P.V.V., Staggenborg, S.A., Ristic, Z. 2008. Response of crops to limited water. In: Royo, C., Nachit, M., DiFonzo, N., Araus, J.L. (eds), Understanding and Modeling Water Stress Effects on Plant Growth Processes: New Challenges. CIHEAM. Zaragoza, Spain. pp. 215-236.

Reddy, N., Reddy, R., Ragimasalawada, M., Sabbavarapu, M.M., Nadoor, S., Patil, J.V. 2014. Detection and validation of stay-green QTL in post-rainy sorghum involving widely adapted cultivar, M35-1 and a popular stay-green genotype B35. BMC Genomics 15:2-16.

Rosenow, D.T., Quisenberry, J.E., Wendt, C.W. 1983. Drought tolerant sorghum and cotton germplasm. Agric. Water Management 7:207-222.

Rostampour, M.F., Yarnia, R.M., Khoee, F., Seghatoleslami, M.J., Moosavi, G.R. 2012. Effect of superb A200 and drought stress on dry matter yield in forage Sorghum. Am.-Eurasian J. Agric. Env. Sci. 12:231-236.

Shamsi, K., Petrosyan, M., Mohammadi, N.G., Haghparast, R. 2010. Evaluation of grain yield and its components in three bread wheat cultivars under drought stress. J. of Animal and Plant Sci. 9:1117-1121.

Songsri, P., Jogloy, S., Holbrook, C.C., Kesmala, T., Vorasoot, N., Akkasaeng, C., Patanothai, A. 2009. Association of root, specific leaf area and SPAD chlorophyll meter reading to water use efficiency of peanut under different available soil water. Agric. Water Management 96:790-798.

Soo-Cheul, Y., Sung-Hwan, C., Zhang, H., Hyo-Chung, P., Chung-Hee, L., Jinjie, L., Jeong-Hoon, Y., ByunWoo, L., Hee-Jong, K., Seo, H.S., Nam-Chon, P. 2007. Quantitative trait loci associated with functional stay-green SNU-SG1 in Rice. Molecules and Cells 24:83-94.

Sticker, F.C., Wearden, S., Pauli, A.W. 1961. Leaf area determination in grain sorghum. Agron. J. 53:187-188.

Taiz, L., Zeiger, E. 2006. Plant Physiology. Sinauer Associates, Inc. Sunderland, UK. pp. 591-602.

Talebi, R., Ensafi, M.H., Baghebani, N., Karami, E., Mohammadi, K. 2013. Physiological responses of chickpea (Cicer arietinum) genotypes to drought stress. Environ. and Exp. Biol. 11:9-15.

Thevar, P.A., Kirkham, M.B., Aiken, R.M., Kofoid, K.D., Xin, Z. 2010. Optimizing water use with high-transpiration-efficiency plants. $19^{\text {th }}$ World Cong. of Soil Science, Soil Solutions for a Changing World. Brisbane, Australia. pp. 804-807.

Xu, W., Subudhi, P.K., Crasta, O.R., Rosenow, D.T., Mullet, J.E., Nguyen, H.T. 2000. Molecular mapping of QTLs conferring stay green in sorghum. Genome 43:461-469.

Yamasaki, S., Dillenburg, L.R. 1999. Measurements of leaf relative water content in Araucaria angustifolia. Revista Brasilleira de Fisiologia Vegetal 11: 69-75.

\section{Electronic Supplementary Material (ESM)}

Electronic Supplementary Material (ESM) associated with this article can be found at the website of CRC at http://www.akademiai.com/content/120427/

Electronic Supplementary Table S1. Correlation among different characters under induced drought stress condition in sorghum 